MPI H-V38-1995

hep-th/9510104

\title{
Tube model for light-front QCD
}

\author{
Brett van de Sande \\ Max Planck Institut für Kernphysik, \\ Postfach 10.39.80, D-69029 Heidelberg, Germany \\ and \\ Matthias Burkardt \\ Physics Department \\ New Mexico State University \\ Las Cruces, NM 88003-0001, U.S.A.
}

\begin{abstract}
We propose the tube model as a first step in solving the bound state problem in light-front QCD. In this approach we neglect transverse variations of the fields, producing a model with $1+1$ dimensional dynamics. We then solve the two, three, and four particle sectors of the model for the case of pure glue SU(3). We study convergence to the continuum limit and various properties of the spectrum.
\end{abstract}

The ultimate goal of light-front field theory is to start with the QCD Lagrangian and, with a minimum of approximation, calculate the hadron spectrum. The basic idea behind this approach is to use Hamiltonian techniques in the coordinate system best suited for relativistic dynamics [1]. We believe that the "tube model" provides a good starting point towards that goal. Simply put, we take full $3+1$ dimensional QCD and restrict our attention to a region that is small in the two transverse directions and is large in the longitudinal dimension, hence the name "tube model." Since the transverse region is small, we neglect any transverse derivatives of the fields as an approximation. The resulting theory is a $1+1$ dimensional gauge theory coupled to adjoint matter. We then employ light-front Hamiltonian techniques to produce the low energy spectrum of the theory. This approach was first explored in the transverse lattice paper of Bardeen, Pearson, and 
Rabinovici [2] (that is, one can think of the tube model as two links on a transverse lattice) and has become increasingly popular in the last few years under the name "dimensional reduction" [3, [4].

The tube model has the virtues of a $1+1$ dimensional theory, in that it is superrenormalizable and computationally tractable, while retaining some of the dynamics of the full theory. Thus, as we demonstrate in this paper, one can hope to understand the behavior of one tube quite thoroughly. The subsequent step will be much more difficult: to couple several of these tubes together to make a full $3+1$ dimensional theory.

In this paper, we study only the pure glue sector of the theory. Of course, an important next step will be to include fermions. Although one may impose antiperiodic boundary conditions on the fermion fields, a consistent formulation that includes fermions must impose periodic boundary conditions on the gauge fields. In anticipation of this situation, we impose periodic boundary conditions for our gauge fields; this will have some important consequences for our numerical results.

The paper is organized as follows. In Sec. 1 1 we discuss (and dismiss) the gauge zero mode. We define the tube model for pure glue in Sec. Q. Then in Secs. 月, 国, and 5 we discuss the two, three, and four particle sectors of the theory. In addition, our technique for measuring parity is demonstrated in Sec. 因. Finally, we discuss some aspects of our results in Sec. 6 .

\section{The gauge zero mode}

Before we begin, we must discuss the "gauge zero mode," $V \equiv \int d x^{-} A_{-}$. As has been noticed by several authors in the context of both light-front [5] and equal time [6] quantization, one cannot set $V=0$ by choice of gauge because it is a genuine dynamical degree of freedom. Only the gauge choice $\partial_{-} A_{-}=0$ is allowed.

What are the effects of $V$ on the tube model particle spectrum? In the Lagrangian, we must replace the longitudinal derivative $\partial_{-}$by the covariant version $\partial_{-}+i g V$. If we regulate the theory by imposing periodic boundary conditions (DLCQ), the longitudinal momentum becomes discrete and we find that the effect of $V$ is to shift momenta by an amount that is less than one longitudinal momentum spacing. Clearly, any direct effect of this mode on ordinary dynamical modes is negligible in the large volume limit $L \rightarrow$ 
$\infty$. However, the zero mode sector of the theory may become qualitatively different.

For instance, some of the zero modes that were otherwise constrained become dynamical. In light-front field theory, equations of motion are typically of the form $\partial_{+}\left(\partial_{-}+i g V\right) \phi=U(\phi)$, where $U(\phi)$ is the potential term. When $V=0$ it is clear why zero modes $\partial_{-} \phi=0$ are not dynamical: they have no conjugate momentum. However, note that when $V$ is nonzero, the associated kinetic term is small (since $V$ is small) relative to the kinetic term of any non-zero mode. Thus, one may just as well treat these zero modes as non-dynamical (that is, as a solution of a constraint equation).

Without the gauge zero mode, Gauß' law requires that all $N^{2}-1$ generators $Q^{a}$ of the gauge group (defined in Eqn. (7)) annihilate physical states. If $V$ is included, only the $N-1$ diagonal generators must annihilate physical states. Consequently, the spectrum contains many more "color singlet" states if $V$ is nonzero. However, one finds that these additional states have eigenvalues that diverge as one takes the continuum limit $L \rightarrow \infty$ and they do not affect the low energy spectrum [7, 8].

Although $V$ may be quite important for understanding the vacuum state itself, its effects on the particle spectrum seem to be small in the type of model we are studying. Thus, we set $V=0$ as a dynamical approximation. It is not clear that $A_{-}$can be so easily neglected in the full $3+1$ dimensional theory. For instance, in $3+1$ dimensions, $A_{-}$has a "proper zero mode," $\int d x^{-} A_{-}$, that is constrained along with a dynamical "global zero mode," $\int d x^{-} d^{2} x^{r} A_{-}$. The effects of the proper zero mode on the particle spectrum could be quite important [9].

\section{The Hamiltonian}

In this section, we define the tube model for $3+1$ dimensional $S U(N)$ gauge theory, introduce our notation, and write out the light-front Hamiltonian. We introduce light front coordinates $x_{\mp}=x^{ \pm}=\left(x^{0} \pm x^{3}\right) / \sqrt{2}$, where $x^{+}$is lightfront "time" and $x^{-}$is the longitudinal spatial coordinate; transverse indices are labeled by $r, s \in\{1,2\}$. Next, we put the system in a box of dimensions $\left(L, L_{\perp}, L_{\perp}\right)$ in the $\left(x^{-}, x^{1}, x^{2}\right)$ directions and impose periodic boundary conditions, or "discretized light-cone quantization" (DLCQ) [1, 10]. As our central assumption, we neglect variations of the fields in the transverse direc- 
tions and set $\partial_{r} A_{\mu}=0$. Since this approximation violates transverse gauge invariance and rotational invariance, we are free to introduce a transverse mass term $\operatorname{tr} A_{r} A_{r}$. As we shall see, inclusion of this term will be necessary for proper renormalization of the model. Thus the Lagrangian is

$$
L_{\text {gauge }}=L_{\perp}^{2} \int d x^{-} \operatorname{tr}\left\{F_{-+} F_{-+}+2 F_{+r} F_{-r}-\frac{1}{2} F_{r s} F_{r s}-\mu_{0}^{2} A_{r} A_{r}\right\},
$$

where

$$
\begin{aligned}
F_{-+} & =\partial_{-} A_{+} \\
F_{-r} & =\partial_{-} A_{r} \\
F_{+r} & =\partial_{+} A_{r}+i g\left[A_{+}, A_{r}\right] \\
F_{r s} & =i g\left[A_{r}, A_{s}\right] .
\end{aligned}
$$

The $A_{+}$field is a constrained degree of freedom. It obeys the constraint equation

$$
-\frac{1}{g} \partial_{-} \partial_{-} A_{+}=J^{+}=i\left[\partial_{-} A_{r}, A_{r}\right],
$$

where $J^{\mu}$ is the conserved current. The conserved charge associated with $J^{\mu}$ is

$$
Q=L_{\perp}^{2} \int d x^{-} J^{+}=i L_{\perp}^{2} \int d x^{-}\left[\partial_{-} A_{r}, A_{r}\right] .
$$

According to Eqn. (6), $Q$ must vanish. In the usual manner, we demand that $Q$ annihilates physical states.

The generators of $S U(N)$ obey the relations $\left[T^{a}, T^{b}\right]=i f^{a b c} T^{c}$ and $\operatorname{tr} T^{a} T^{b}=\delta^{a b} / 2$. Writing $A_{r}=A_{r}^{a} T^{a}$, the momentum conjugate to $A_{r}^{a}$ is $\partial_{-} A_{r}^{a}$. Thus the zero mode of $A_{r}^{a}$ has no conjugate momentum and is not a true dynamical degree of freedom. For the rest of this discussion, we will drop the zero mode associated with $A_{r}$. Next, we construct the helicity operator $\mathcal{J}_{z}$ which generates rotations in the $\left(x^{1}, x^{2}\right)$ plane, the longitudinal momentum operator $P^{+}$, and the Hamiltonian $P^{-}$:

$$
\begin{aligned}
\mathcal{J}_{z} & =-4 L_{\perp}^{2} \int d x^{-} \operatorname{tr} A_{1} \partial_{-} A_{2} \\
P^{+} & =2 L_{\perp}^{2} \int d x^{-} \operatorname{tr} \partial_{-} A_{r} \partial_{-} A_{r} \\
P^{-} & =L_{\perp}^{2} \int d x^{-} \operatorname{tr}\left\{-g^{2} J^{+} \frac{1}{\left(\partial_{-}\right)^{2}} J^{+}+\mu_{0}^{2} A_{r} A_{r}-\frac{g^{2}}{2}\left[A_{r}, A_{s}\right]^{2}\right\} .
\end{aligned}
$$


The inverse derivative operator is well-defined in this case since $J^{+}$has no zero mode in the space of physical states.

Now we are ready to quantize the theory. We can expand $A_{r}$ in terms of creation and annihilation operators:

$$
A_{r}^{a}=\frac{1}{L_{\perp} \sqrt{4 \pi}} \sum_{\lambda= \pm 1} \sum_{k=1}^{\Lambda} \frac{1}{\sqrt{k}}\left(a_{k, \lambda}^{a} \epsilon_{r}^{-\lambda} e^{-2 \pi i k x^{-} / L}+a_{k, \lambda}^{a}{ }^{\dagger} \epsilon_{r}^{\lambda} e^{2 \pi i k x^{-} / L}\right) .
$$

The creation/annihilation operators obey the usual commutation relations,

$$
\begin{aligned}
{\left[a_{k, \lambda}^{a}, a_{l, \lambda^{\prime}}^{b}\right] } & =\delta^{a, b} \delta_{k, l} \delta_{\lambda, \lambda^{\prime}} \\
{\left[a_{k, \lambda}^{a}, a_{l, \lambda^{\prime}}^{b}\right] } & =\left[a_{k, \lambda^{\dagger}}^{a}, a_{l, \lambda^{\prime}}^{b}\right]=0 .
\end{aligned}
$$

We define the polarization tensor $\epsilon_{r}^{\lambda}: \epsilon_{1}^{ \pm 1}=1 / \sqrt{2}$ and $\epsilon_{2}^{ \pm 1}= \pm i / \sqrt{2}$. Using the creation/annihilation operators and normal ordering, we find

$$
\begin{array}{r}
\mathcal{J}_{z}=\sum_{k=1}^{\Lambda} \lambda a_{k, \lambda}^{a}{ }^{\dagger} a_{k, \lambda}^{a} \\
P^{+}=\frac{2 \pi}{L} \sum_{k} k a_{k, \lambda}^{a}{ }^{\dagger} a_{k, \lambda}^{a} \\
Q^{a}=-i \sum_{k=1}^{\Lambda} f^{a b c} a_{k, \lambda}^{b}{ }^{\dagger} a_{k, \lambda}^{c} .
\end{array}
$$

Let us examine the Hamiltonian $P^{-}$in more detail. From the first two terms in Eqn. (10) we obtain the four instantaneous interactions, the "selfinduced inertias," and the mass term,

$$
\begin{aligned}
& P_{1}^{-}=\frac{g^{2} L}{16 \pi^{2} L_{\perp}^{2}} \sum_{n^{\prime}, m^{\prime}, n, m} \frac{\sqrt{n n^{\prime}} \delta_{m+n, m^{\prime}+n^{\prime}} f^{a b c} f^{a^{\prime} b^{\prime} c}}{\sqrt{m m^{\prime}}(m+n)^{2}} \\
& \cdot a_{n,-\lambda}^{a} a_{m, \lambda}^{b} a_{n^{\prime}, \lambda^{\prime}}^{a^{\prime}} a_{m^{\prime},-\lambda^{\prime}}^{b^{\prime}} \\
& P_{2}^{-}=\frac{g^{2} L}{16 \pi^{2} L_{\perp}^{2}} \sum_{n^{\prime}, m^{\prime}, n, m} \frac{(m+n) \sqrt{n^{\prime}} \delta_{n, m+m^{\prime}+n^{\prime}} f^{a b c} f^{a^{\prime} b^{\prime} c}}{\sqrt{m n m^{\prime}}\left(m^{\prime}+n^{\prime}\right)^{2}} \\
& \cdot a_{n, \lambda}^{a} a_{n^{\prime}, \lambda^{\prime}}^{a^{\prime}} a_{m^{\prime},-\lambda^{\prime}}^{b^{\prime}} a_{m, \lambda}^{b} \\
& P_{3}^{-}=-\frac{g^{2} L}{16 \pi^{2} L_{\perp}^{2}} \sum_{n^{\prime}, m^{\prime}, n, m} \frac{\sqrt{n}\left(m^{\prime}+n^{\prime}\right) \delta_{m+n+n^{\prime}, m^{\prime}}}{\sqrt{m^{\prime} n^{\prime} m}(m+n)^{2}}
\end{aligned}
$$




$$
\begin{aligned}
& \cdot f^{a b c} f^{a^{\prime} b^{\prime} c} a_{n^{\prime}, \lambda^{\prime}}^{a^{\prime}} a_{n,-\lambda}^{a}{ }^{\dagger} a_{m, \lambda}^{b} a^{\dagger} a_{m^{\prime}, \lambda^{\prime}}^{b^{\prime}} \\
& P_{4}^{-}=-\frac{g^{2} L}{16 \pi^{2} L_{\perp}^{2}} \sum_{n^{\prime}, m^{\prime}, n, m, k>0} \frac{(m+n)\left(m^{\prime}+n^{\prime}\right)}{\sqrt{m n m^{\prime} n^{\prime}} k^{2}} \\
& \cdot \delta_{n, k+m} \delta_{m+m^{\prime}, n+n^{\prime}} f^{a b c} f^{a^{\prime} b^{\prime} c} a_{n^{\prime}, \lambda^{\prime}}^{a^{\prime}} a_{n, \lambda}^{a} a_{m^{\prime}, \lambda^{\prime}}^{b^{\prime}} a_{m, \lambda}^{b} \\
& P_{5}^{-}=\frac{g^{2} L N}{32 \pi^{2} L_{\perp}^{2}} \sum_{m} f(m) a_{m, \lambda}^{b} a_{m, \lambda}^{b} \\
& P_{6}^{-}=\frac{L \mu^{2}}{4 \pi} \sum_{k} \frac{a_{k, \lambda}^{c} a_{k, \lambda}^{c}}{k} .
\end{aligned}
$$

The self-induced inertia $P_{5}^{-}$is obtained by bringing the instantaneous interactions in Eqn. (10) into normal ordered form, Eqns. (17) and (20). Before mass renormalization, the associated function $f(m)$ is

$$
f(m)=8 \sum_{k=1}^{m-1} \frac{1}{k^{2}}+\frac{2}{m}\left(\ln (\Lambda)+\gamma_{E}\right)+\frac{3}{m^{2}}+O(1 / \Lambda) .
$$

We choose our mass renormalization such that the logarithmic divergence is removed

$$
f(m)=8 \sum_{k=1}^{m-1} \frac{1}{k^{2}}+\frac{3}{m^{2}}+\frac{8}{m} \approx \frac{4 \pi^{2}}{3} .
$$

Next, we can write out terms associated with $\left[A_{r}, A_{s}\right]^{2}$. Any contracted terms have the same operator structure as the mass term and are removed by mass renormalization.

$$
\begin{aligned}
P_{7}^{-} & =\frac{g^{2} L}{16 \pi^{2} L_{\perp}^{2}} \sum_{k, l, m} \frac{f^{a b c} f^{a^{\prime} b^{\prime} c}}{\sqrt{k \operatorname{lm}(k+l+m)}} \\
P_{8}^{-} & =\frac{g^{2} L}{16 \pi^{2} L_{\perp}^{2}} \sum_{k, l, m} \frac{f^{a b c} f^{a} a^{a^{\prime} b^{\prime} c}}{\sqrt{k \operatorname{lm}(k+l+m)}} \\
P_{9}^{-} & =\frac{g^{2} L}{32 \pi^{2} L_{\perp}^{2}} \sum_{k, l, m, n}^{b} \frac{\delta_{m, \lambda}^{b_{m, n}} a_{l, \lambda^{\prime}}^{a^{\prime}} a^{\dagger} a_{l, \lambda^{\prime}}^{a^{\prime}} f^{b^{\prime}} f^{b^{\prime}} f^{a^{\prime} b^{\prime} c}}{\sqrt{k l m n}}
\end{aligned}
$$




$$
\begin{aligned}
& \cdot a_{k, \lambda^{\prime}}^{a} a_{l, \lambda}^{b}{ }^{\dagger} a_{m, \lambda^{\prime}}^{a^{\prime}} a_{n, \lambda}^{b^{\prime}} \\
& P_{10}^{-}=\frac{g^{2} L}{32 \pi^{2} L_{\perp}^{2}} \sum_{k, l, m, n} \frac{\delta_{l+n, k+m} f^{a b c} f^{a^{\prime} b^{\prime} c}}{\sqrt{k \operatorname{lm} n}} \\
& \cdot a_{k, \lambda^{\prime}}^{a} a_{m,-\lambda^{\prime}}^{a^{\prime}} a_{l, \lambda}^{b} a_{n,-\lambda}^{b^{\prime}} \\
& P_{11}^{-}=\frac{g^{2} L}{32 \pi^{2} L_{\perp}^{2}} \sum_{k, l, m, n} \frac{\delta_{l+m, k+n} f^{a b c} f^{a^{\prime} b^{\prime} c}}{\sqrt{k l m n}} \\
& \cdot a_{k, \lambda}^{a}{ }^{\dagger} a_{n, \lambda^{\prime}}^{b^{\prime}}{ }^{\dagger} a_{l, \lambda^{\prime}}^{b} a_{m, \lambda}^{a^{\prime}} .
\end{aligned}
$$

The relation between the bare mass $\mu_{0}$ and the renormalized mass $\mu$ in Eqn. (22) is

$$
\mu_{0}^{2}=\mu^{2}+\frac{g^{2} N}{4 \pi L_{\perp}^{2}}-\frac{g^{2} N}{2 \pi L_{\perp}^{2}} \sum_{m=1}^{\Lambda} \frac{1}{m} .
$$

In the subsequent numerical work, we choose

$$
1=\frac{g^{2} N}{4 \pi L_{\perp}^{2}}
$$

which sets the mass scale. For the three and four particle truncations where we need to specify the number of colors we set $N=3$. Also, we define $M^{2}$ as the eigenvalue of the (invariant mass) ${ }^{2}$ operator $P_{\mu} P^{\mu}=2 P^{+} P^{-}$. If we define the integer valued "harmonic resolution" $K=L P^{+} /(2 \pi)$, the continuum limit is achieved by taking $\lim K \rightarrow \infty$ for fixed $P^{+}$. States are classified by the conserved quantities: helicity $\mathcal{J}_{z}$, particle number mod 2 , charge conjugation $\mathcal{C}$, and parity $\mathcal{P}$. We will often use the notation $\mathcal{J}_{z}{ }^{\mathcal{P C}}$. Under charge conjugation, gauge fields transform as $\left(A_{r}\right)_{i j} \rightarrow-\left(A_{r}\right)_{j i}$ and under parity, they transform as $A_{r}\left(x^{+}, x^{-}\right) \rightarrow-A_{r}\left(x^{-}, x^{+}\right)$(actually, $P^{+}$ eigenstates are eigenstates of parity times a longitudinal boost). Note that we use the definition of parity appropriate for $3+1$ dimensions. Our method for measuring parity will be explained in more detail in Section 1 .

\section{Two particles}

The low energy states in this type of model have, to a good approximation, definite particle number [3]. Thus, it is useful to study the spectrum by 


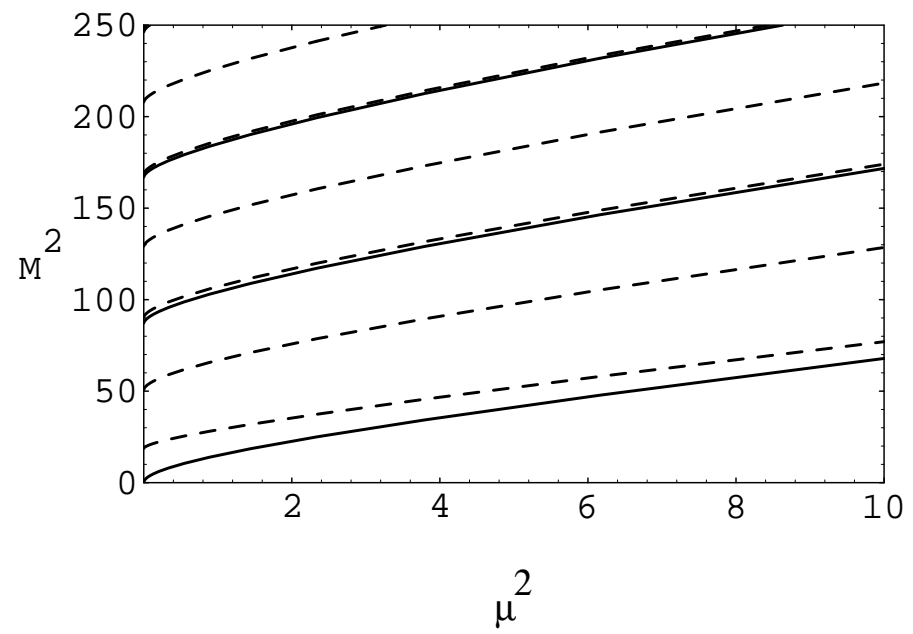

Figure 1: Two particle spectrum vs $\mu^{2}$ for $\mathcal{J}_{z}=0$ (dashed curves) and $\mathcal{J}_{z}=2$ (solid curves) using the polynomial wave function basis calculation discussed at the end of Sec. 国. For $\mathcal{J}_{z}=0$, the states alternate between even and odd parity.

applying a truncation in particle number. In this section, we apply a two particle truncation and study the bound state equation using DLCQ and a polynomial wave function basis in the continuum limit.

Two particle eigenstates have the general form

$$
\sum_{j=1}^{K-1} \phi_{j} a_{j, \lambda}^{a} \dagger^{\dagger} a_{K-j, \lambda^{\prime}}^{a}|0\rangle
$$

where the total helicity is $\mathcal{J}_{z}=\lambda+\lambda^{\prime}$. These states are even under charge conjugation $\mathcal{C}=1$. For $\mathcal{J}_{z}=2$, the wavefunction must be even, $\phi_{j}=\phi_{K-j}$, since the particles are identical $\left(\mathcal{J}_{z}^{\mathcal{P C}}=2^{++}\right)$, while they can be even or odd for $\mathcal{J}_{z}=0\left(\mathcal{J}_{z}{ }^{\mathcal{P C}}=0^{++}\right.$or $\left.0^{-+}\right)$. The spectrum as a function of mass is shown in Fig. 1. The states are roughly evenly spaced due to the linear potential generated by the instantaneous interactions $P_{1}^{-}+\cdots+P_{4}^{-}$. In addition, for $\mu^{2}=0$, it is evident that the ground state is massless in the continuum limit. The corresponding wavefunctions at $\mu^{2}=0$ are roughly sines and cosines as shown in Figs. 2 and 3 .

Taking the continuum limit $K \rightarrow \infty$, one can convert the bound state 


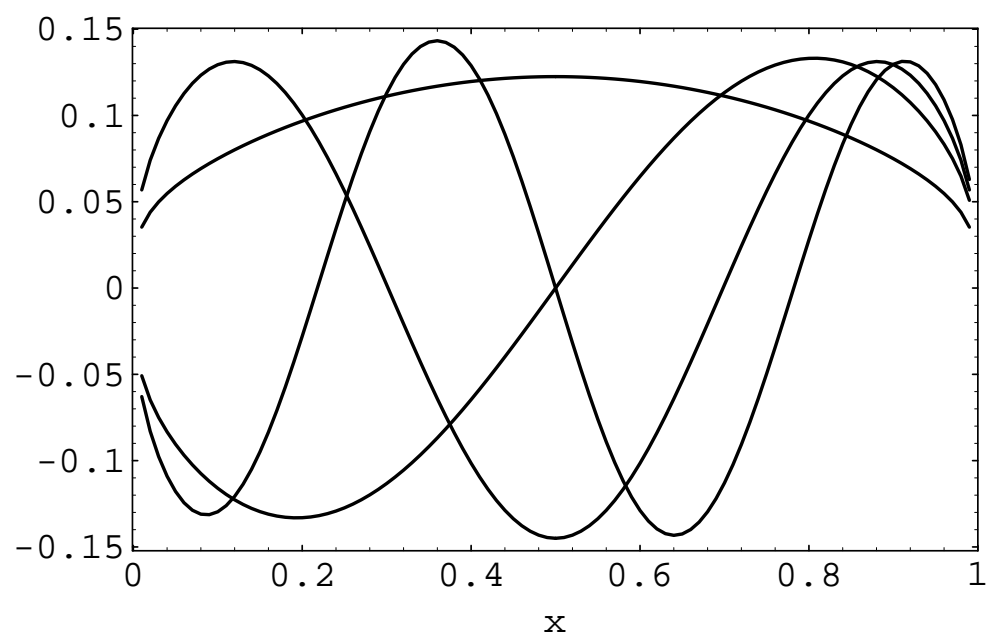

Figure 2: Lowest eigenfunctions $\phi_{j}$ vs momentum fraction $x=j / K$ for $\mathcal{J}_{z}=0, \mu^{2}=0$, and $K=100$. Wavefunctions are either even or odd, depending on the parity of the state.

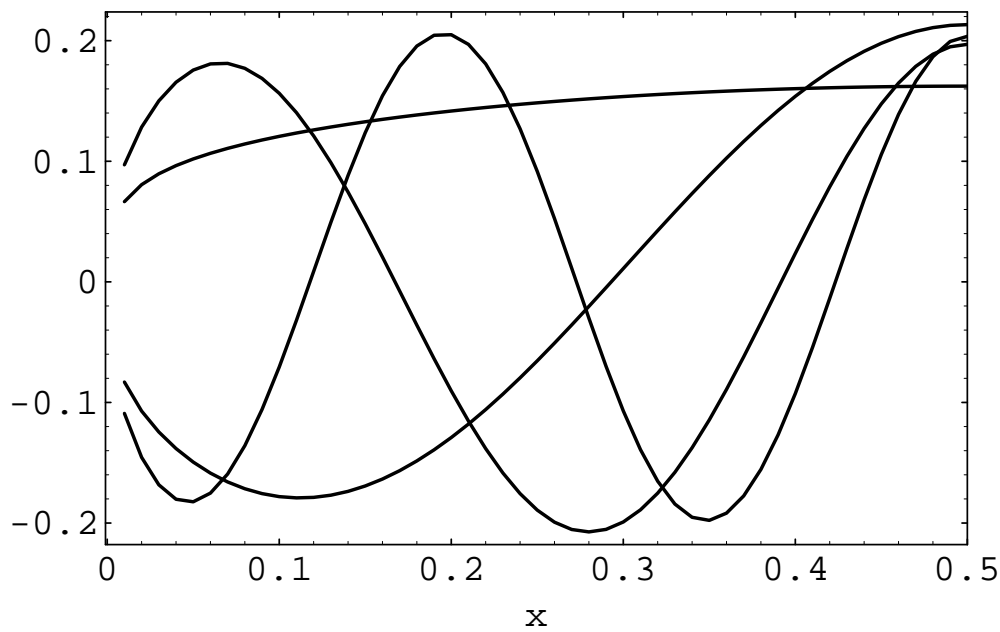

Figure 3: Lowest eigenfunctions $\phi_{j}$ vs momentum fraction $x=j / K$ for $\mathcal{J}_{z}=2, \mu^{2}=0$, and $K=100$. The shape of the lowest wavefunction is a finite $K$ artifact; it becomes constant in the continuum limit $K \rightarrow \infty$. 
equation into an integral equation

$$
\begin{aligned}
M^{2} \phi(x)= & \mu^{2} \phi(x)\left(\frac{1}{x}+\frac{1}{1-x}\right) \\
& -\frac{g^{2} N}{4 \pi L_{\perp}^{2}} \int \frac{d y(x+y)(2-x-y)}{(x-y)^{2} \sqrt{x(1-x) y(1-y)}} \phi(y) \\
& \mp \frac{g^{2} N}{4 \pi L_{\perp}^{2}} \int \frac{d y}{\sqrt{x(1-x) y(1-y)}} \phi(y)+O(1 / K) .
\end{aligned}
$$

where $x, y \in(0,1)$ and we identify $\phi(i / K)$ with $\phi_{i}$ in the discrete case. For $\mathcal{J}_{z}=2$, the coefficient of the last term of (33) is negative and $\phi(x)=$ $\phi(1-x)$. For $\mathcal{J}_{z}=0$, the coefficient of the last term is positive and $\phi(x)=$ $\pm \phi(1-x)$. The renormalization prescription we introduced for the discrete case Eqn. (30) is equivalent to using a principal-value prescription for the pole in the first integral of Eqn. (33). One finds that $\phi(x)=1, M^{2}=$ 0 is an exact solution for $\mathcal{J}_{z}=2$ and $\mu^{2}=0$, confirming our numerical results. Below this mass, the spectrum becomes tachyonic independent of the $\left[A_{r}, A_{s}\right]^{2}$ interaction. Also, we can repeat 't Hooft's end-point analysis [2, 12] for this state. Assume that the wavefunction has power law behavior $\phi(x) \propto x^{\beta}$ near the endpoint $x=0$. To leading order in $x$, the bound state equation becomes

$$
0=\mu^{2} x^{\beta-1}-\frac{g^{2} N}{2 \pi L_{\perp}^{2}} \int_{0}^{1} \frac{d y(x+y) y^{\beta-1 / 2}}{(x-y)^{2} \sqrt{x}},
$$

which yields the relation

$$
\mu^{2}=\frac{g^{2} N}{\pi L_{\perp}^{2}} \pi \beta \tan (\pi \beta), \quad 0 \leq \beta \leq 1 / 2 .
$$

Using this expression for the endpoint behavior, one can also show that $M^{2}$ depends linearly on $\mu$ near $\mu^{2}=0$ :

$$
\lim _{\mu^{2} \rightarrow 0} M^{2}=4 \pi \mu \sqrt{\frac{g^{2} N}{4 \pi L_{\perp}^{2}}} .
$$

Next, let us examine the convergence to the continuum limit for DLCQ. If we plot the spectrum as a function of harmonic resolution, we seem to find 


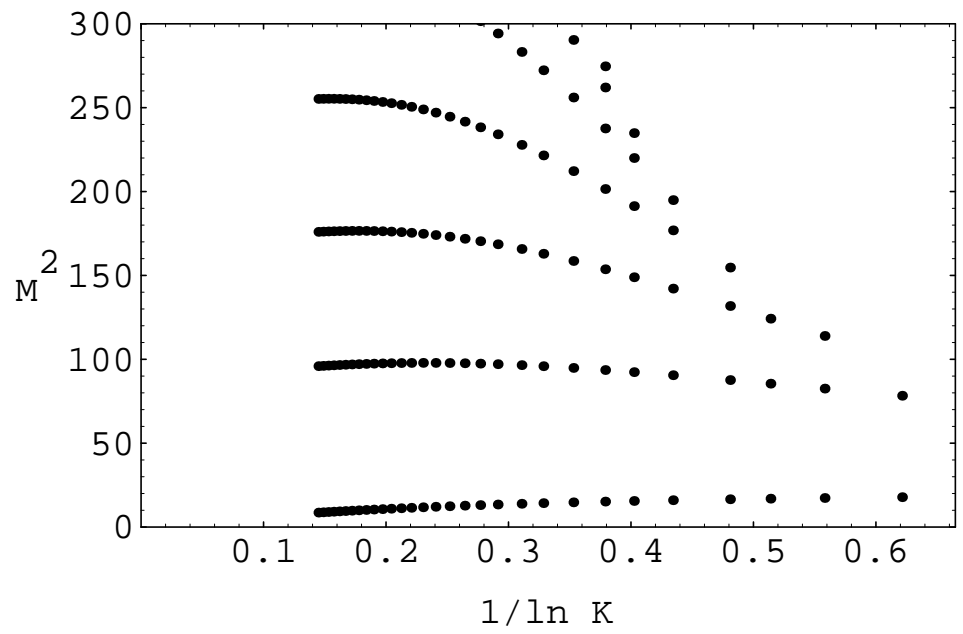

Figure 4: Two particle spectrum for helicity $2, \mu^{2}=0$, as a function of harmonic resolution. According to this plot, convergence to the continuum limit seems to be quite good.

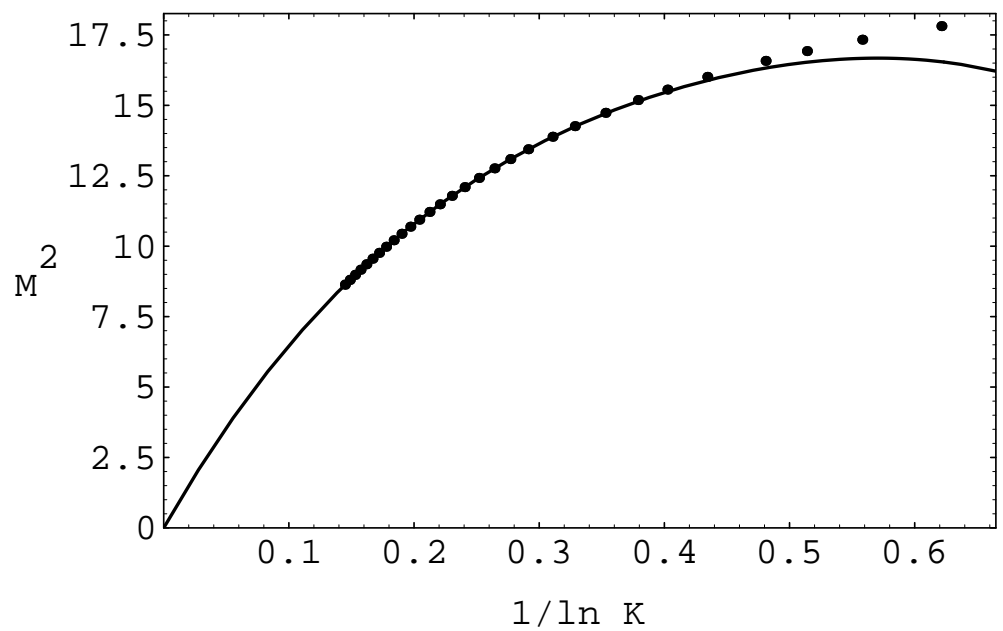

Figure 5: Ground state eigenvalue for helicity 2, $\mu^{2}=0$ as a function of harmonic resolution together with a numerical fit to $78.08 / \ln K-$ $150.52 /(\ln K)^{2}+180.21 /(\ln K)^{3}+\ldots$ In fact, convergence to the continuum limit is quite poor due to our having neglected the zero mode. 


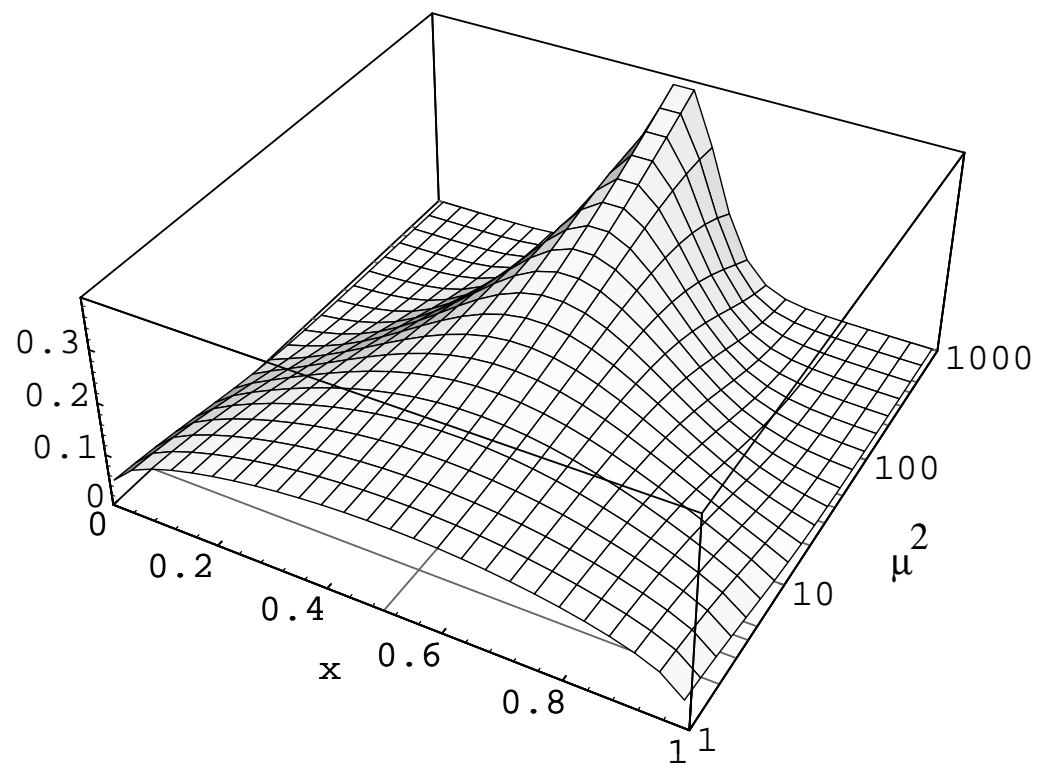

Figure 6: Lowest eigenfunction vs $\mu^{2}$ for $\mathcal{J}_{z}=2$ and $K=102$. The wavefunction is constant at $\mu^{2}=0$ and becomes a delta function at $\mu^{2}=\infty$.

quick convergence as shown in Fig. 1. However, if we examine the ground state more closely in Fig. 5, we see that its eigenvalue in fact converges quite slowly. The same is true for the eigenfunctions. Let us take a variational ansatz for the wavefunction: $\phi_{j}=j^{\beta}(K-j)^{\beta}$. For $\mathcal{J}_{z}=2, \mu^{2}=0$, we find that this ansatz is a very good approximation of the ground state wavefunction for all $K\left(M^{2}\right.$ eigenvalues differ by only about 0.002$)$ and that the wavefunction is constant $(\beta \rightarrow 0)$ in the $K \rightarrow \infty$ limit:

$$
\beta \approx 1.71 / \ln K-2.95 /(\ln K)^{2}+5.49 /(\ln K)^{3}+\ldots .
$$

This explains the shape of the lowest eigenfunction in Figs. 3 and 6 .

This slow convergence is due to our having treated the zero mode sector of the theory poorly: we have simply removed the zero momentum mode from the theory. In terms of the continuum formulation, Eqn. (33), neglecting the zero mode is equivalent to introducing cutoffs in momenta. That is,

$$
\varepsilon \leq x, y \leq 1-\varepsilon,
$$




$$
\varepsilon \leq|x-y|
$$

where we identify $\varepsilon \approx 2 / K$. In this case the pole associated with the first integral is removed by adding a term of the form

$$
\frac{2 g^{2} N}{\pi L_{\perp}^{2}} \frac{\phi(x)}{\varepsilon}
$$

to the right hand side of (33). Using the variational ansatz $\phi(x)=x^{\beta}(1-x)^{\beta}$, for $\mathcal{J}_{z}=2, \mu^{2}=0$, we find the same behavior that we did in the discrete case:

$$
\begin{aligned}
\beta & \approx-\frac{1.838}{\ln \varepsilon}-\frac{3.399}{(\ln \varepsilon)^{2}}-\frac{12.611}{(\ln \varepsilon)^{3}}+\ldots \\
\left\langle\phi\left|P_{\mu} P^{\mu}\right| \phi\right\rangle & \approx-\frac{85.15}{\ln \varepsilon}-\frac{197.96}{(\ln \varepsilon)^{2}}-\frac{518.98}{(\ln \varepsilon)^{3}}+\ldots
\end{aligned}
$$

This slow convergence problem gradually disappears as $\mu^{2}$ is increased since the amplitude of the wavefunctions becomes small near $x=0$ and 1 ; see Fig. 6.

In light of the problems associated with DLCQ, we introduce a polynomial wave function basis [2] to calculate the spectrum using the continuum limit of the bound state equation (33). We assume the wavefunction is of the form $\phi(x)=x^{\beta}(1-x)^{\beta} P_{n}(x)$, where $P_{n}(x)$ is a polynomial of order $n$ and $\beta$ is given by Eqn. (35). This method is extremely accurate. For example, with only five polynomials in the basis we obtain a relative error of between $10^{-4}$ $\left(\mu^{2} \approx 0\right)$ and $10^{-3}\left(\mu^{2} \approx 10\right)$ for the ground state eigenvalue.

\section{Three particles}

For the three and four particle truncations, we use only the DLCQ approach since it would be difficult to implement a wave function basis. Unfortunately, the slow convergence problem that we saw in the two particle truncation will affect the three and four particle spectra as well. In fact, the results we present for finite $K$ and $\mu^{2}=0$ better reflect the behavior of the model for small but nonzero $\mu^{2}$ in the continuum limit. That is, the dominant finite $K$ 


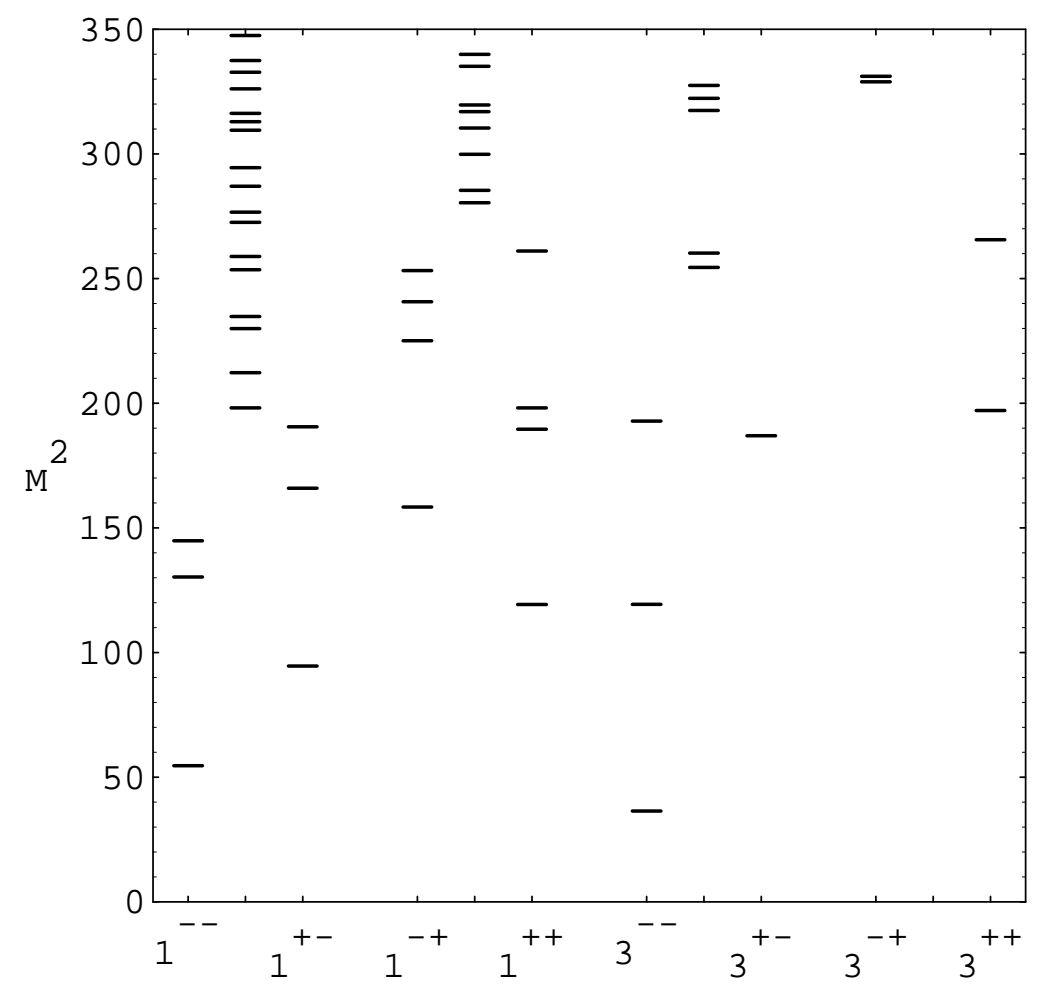

Figure 7: Three particle spectrum for various values of $\mathcal{J}_{z}^{\mathcal{P C}}$ where $\mu^{2}=0$ and $K=25\left(\mathcal{J}_{z}=1\right)$ or $K=35\left(\mathcal{J}_{z}=3\right)$. States whose parity could not be determined are placed midway between the corresponding $\mathcal{J}_{z}{ }^{+\mathcal{C}}$ and $\mathcal{J}_{z}{ }^{-\mathcal{C}}$ columns. 
effects can be absorbed into a finite mass renormalization. In any case, the numbers we present should not be considered to be precise.

The three particle spectrum is shown in Fig. 7. Color singlet states are constructed from the symmetric tensor $d_{a b c}(\mathcal{C}=-1)$ and the antisymmetric tensor $f_{a b c}(\mathcal{C}=1)$ [13]. The spectrum behaves much in the same way that atomic spectra do: the greater the symmetry of the wavefunction, the lower the energy of the state. Thus, the $\mathcal{J}_{z}=3$ sector has lower energy than the $\mathcal{J}_{z}=1$ sector; $\mathcal{C}=-1\left(d_{a b c}\right)$ states have lower energy than $\mathcal{C}=1\left(f_{a b c}\right)$ states; and $\mathcal{P C}=1$ states have lower energy than $\mathcal{P C}=-1$ states. In fact, we find the same basic behavior in the two particle sector and, for the most part, in the four particle sector as well.

Next, we discuss our method for measuring parity using the three particle spectrum as an example. Since the parity operator exchanges $x^{+}$and $x^{-}$, it is a dynamical operator in light-front quantization and is, in general, difficult to construct. In addition, parity is broken by our DLCQ cutoff and can only be recovered in the continuum limit. (We apply periodic boundary conditions in the $x^{-}$direction, but not in the $x^{+}$direction.) However, in a theory without fermions we expect that parity will be restored automatically in the continuum limit. To determine the parity of a state, we first determine its parity for finite but large $\mu^{2}$ using Hornbostel's parity transform for free fields [11]; we then decrease the mass to the value of interest.

Three particle eigenstates have the general form:

$$
\left(f_{a b c} \text { or } d_{a b c}\right) \sum_{l \leq m} \sum_{n} \delta_{K, l+m+n} \phi_{l m} a_{l, 1}^{a}{ }^{\dagger} a_{m, 1}^{b}{ }^{\dagger} a_{n,-1}^{c}{ }^{\dagger}|0\rangle, \quad \mathcal{J}_{z}=1
$$

and

$$
\left(f_{a b c} \text { or } d_{a b c}\right) \sum_{l \leq m \leq n} \delta_{K, l+m+n} \phi_{l m} a_{l, 1}^{a} a^{\dagger} a_{m, 1}^{b} a_{n, 1}^{c}|0\rangle, \quad \mathcal{J}_{z}=3 .
$$

In Ref. [11] Hornbostel introduces a parity transform (times a boost) that is valid for free fields. In general, a particle with longitudinal momentum fraction $x_{i}$ transforms as

$$
x_{i} \rightarrow \tilde{x}_{i}=\frac{1}{x_{i} \sum_{j} \frac{1}{x_{j}}} .
$$




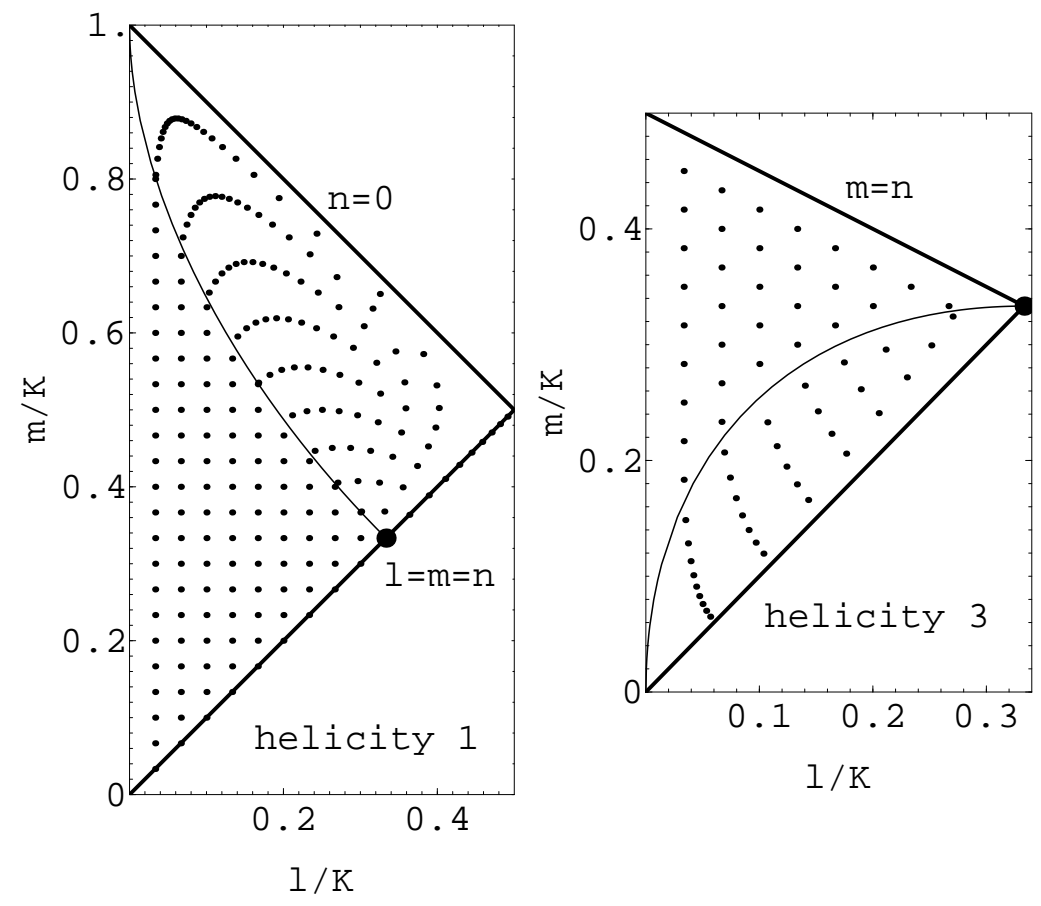

Figure 8: Hornbostel's parity transform applied to three particle wavefunctions in Eqns. (43) and (44). The transform maps each momentum combination $(l, m)$ into another momentum combination $(\tilde{l}, \tilde{m})$. Dots below the invariant curve, Eqns. (46) and (47), are mapped onto dots above the curve. 

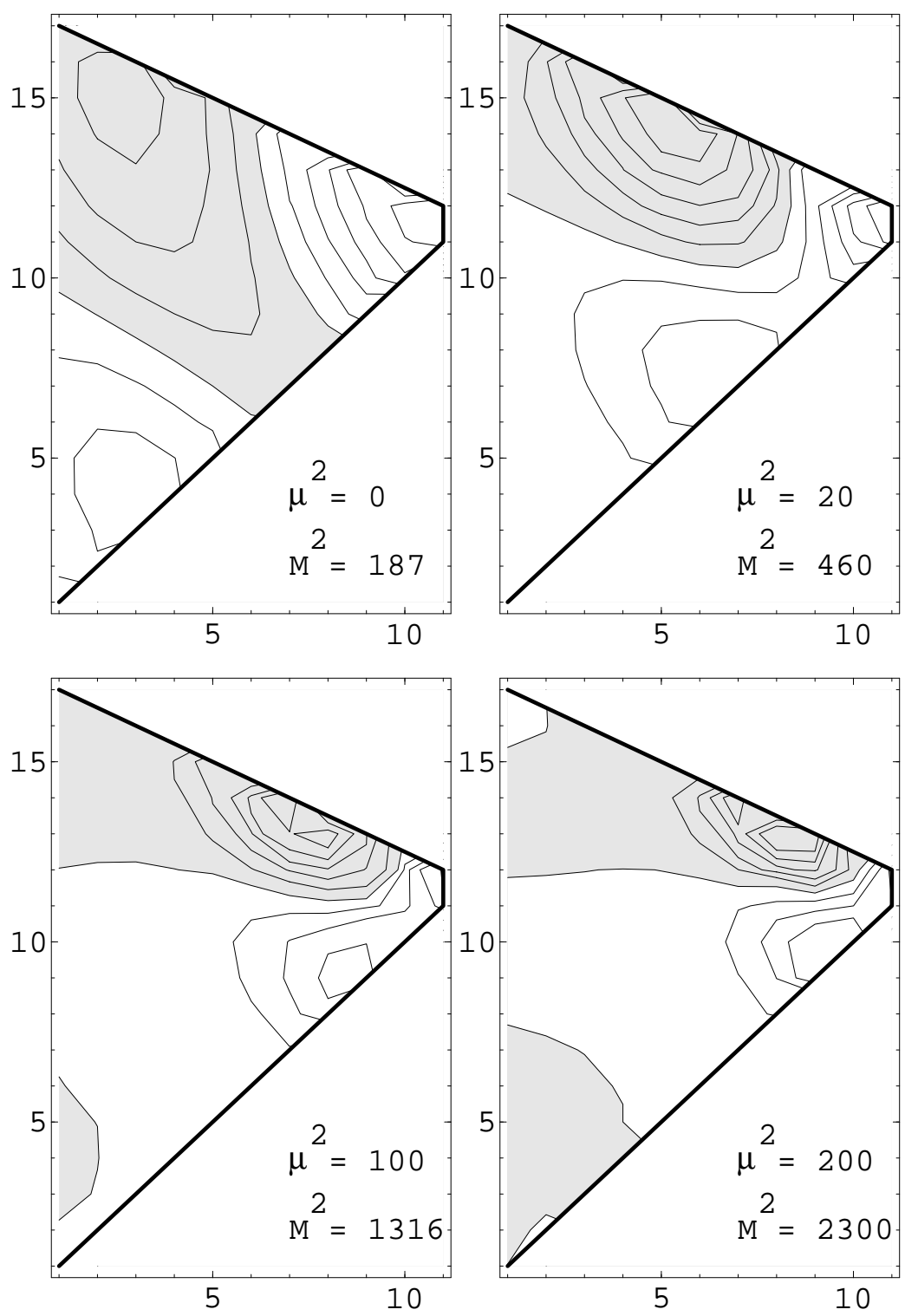

Figure 9: Contour plots of the wavefunction $\phi_{l m}$ vs $l$ and $m$ for the lowest $\mathcal{J}_{z}^{\mathcal{P C}}=3^{+-}$state and various values of $\mu^{2}$. Regions of negative amplitude are shaded gray. Comparing this plot with Fig. 8, we see that the wavefunction becomes an approximate eigenstate of Hornbostel's parity operator as $\mu^{2}$ is increased. 
In Fig. 8 we apply this transformation to Eqns. (43) and (44). In each case there is a curve that is invariant under the transformation:

$$
m=K-\frac{l}{2}-\sqrt{l\left(K-\frac{3}{4} l\right)}, \quad \mathcal{J}_{z}=1
$$

and

$$
m=\sqrt{l\left(K-\frac{3}{4} l\right)}-\frac{l}{2}, \quad \mathcal{J}_{z}=3 .
$$

For free fields, states whose wave functions $\phi_{l m}$ are even (odd) under this transform have even (odd) $\mathcal{P C}$.

Since we are studying an interacting theory, we do not expect Hornbostel's parity operator to work very well for small $\mu^{2}$. This is, in fact, the case. On the other hand, as we increase $\mu^{2}$, we see that the eigenfunctions tend to become approximate eigenstates of Hornbostel's parity operator. Thus, we determine the parity of a state by applying Hornbostel's parity operator to the state for large $\mu^{2}$. However, in the large mass limit $\mu^{2} \rightarrow \infty$, finite $K$ effects become important: eigenstates become states of definite momenta and the expectation value of Hornbostel's parity operator vanishes. Fortunately, there is a mass region $200<\mu^{2}<1000$ where Hornbostel's parity operator does work fairly well. For example, if we compare Figs. 8 and 9, we see that, as $\mu^{2}$ increases, the state becomes more approximately an eigenstate of Hornbostel's parity operator.

Finally, we examine the polarized structure functions for the lowest $1^{--}$ state in Figs. 10 and 11. We see that the antiparallel gluon has a somewhat harder momentum distribution than the two parallel gluons. This is the opposite of what one would normally expect on the basis of helicity retention [14]. Physically, this result is related to the fact that the $2^{++}$glueball is lighter than the $0^{++}$glueball. The reason is the following: If one thinks of the $1^{--}$glueball as a gluon-digluon bound state, then the digluon is lighter when it is in a $2^{++}$state than when it is in a $0^{++}$state. The $\mathcal{J}_{z}=2$ digluon requires an antiparallel third gluon to yield total $\mathcal{J}_{z}=1$, while the $\mathcal{J}_{z}=0$ digluon requires a parallel gluon. Since the $\mathcal{J}_{z}=2$ digluon is lighter, it carries less momentum fraction than the $\mathcal{J}_{z}=0$ digluon, and hence the antiparallel third gluon tends to be shifted towards larger momenta than the parallel third gluon. Of course, this argument oversimplifies the situation somewhat since 


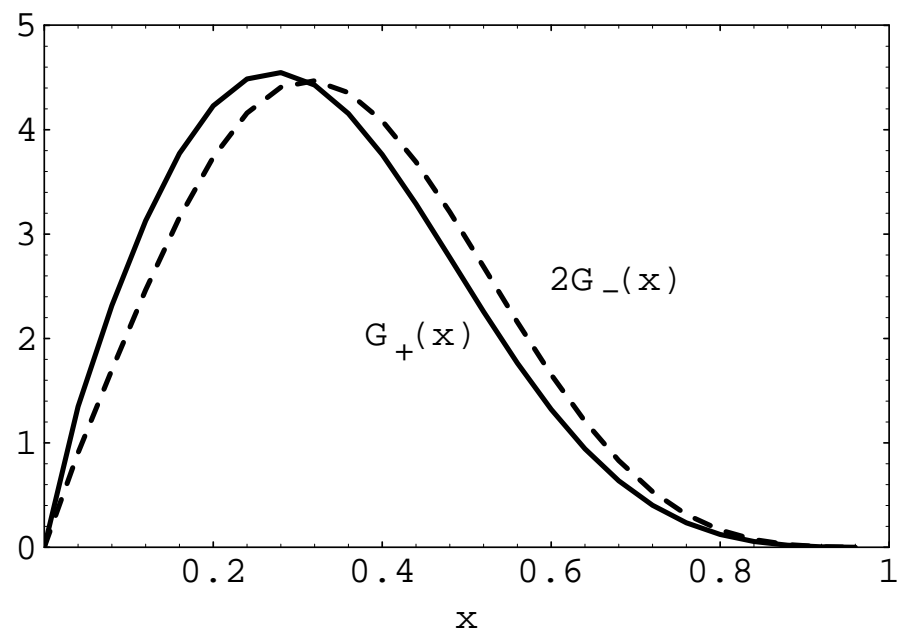

Figure 10: Polarized structure functions of the lowest $1^{--}$state for $K=25$ and $\mu^{2}=0$.

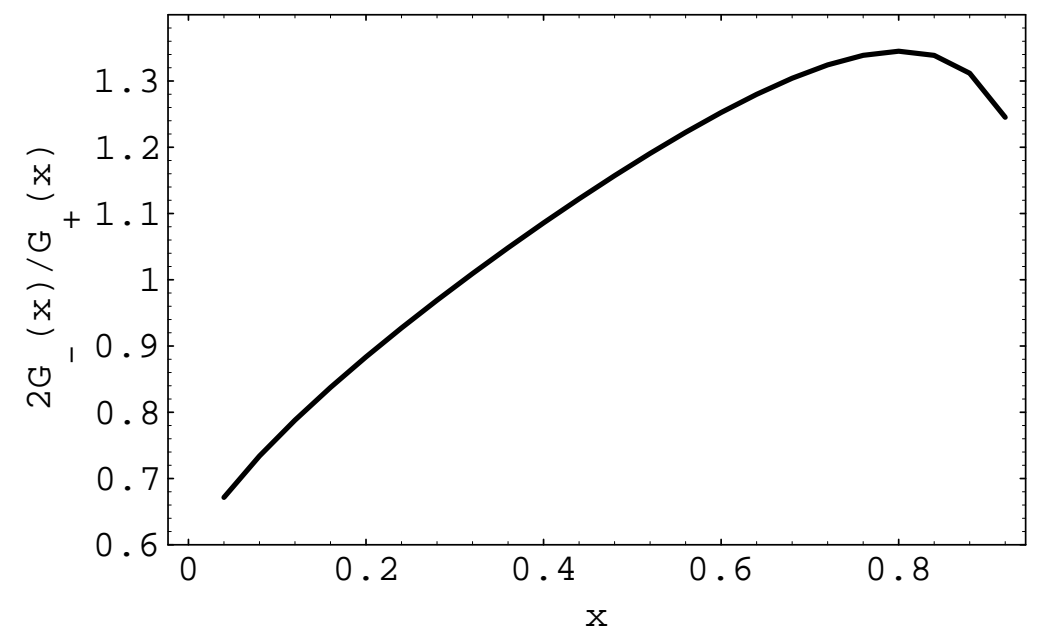

Figure 11: Ratio of the structure functions in Fig. 10. The antiparallel gluon distribution $G_{-}(x)$ is somewhat "harder" than the parallel gluon distribution $G_{+}(x)$. 
there is spin dependence in the interaction between the "digluon" and the "third" gluon. Furthermore, since the gluons are indistinguishable one cannot really separate them into a "digluon" and a "third gluon". Nevertheless, our main point should still be correct: that there is a connection between the $2^{++}$ glueball being lighter than the $0^{++}$glueball and having antiparallel gluons that are "harder" than parallel gluons.

\section{$5 \quad$ Four particles}

When one includes Fock components with a maximum of four gluons, several new phenomena occur: states that were present already within the two particle truncation mix with states that have four particles and new states appear that have little or no two particle content. Although the number of colors $N$ must be specified for the three particle truncation, the four particle sector is the first place where the value of $N$ can have an important effect on the spectrum. The spectrum is shown in Fig. 12; values of $M^{2}$ quoted later in this section are based on this spectrum.

The $\mathcal{C}=-1$ sector contains states that are rather high in energy. These states of course have no two particle content. It is rather puzzling that the lowest state in each $\mathcal{C}=-1$ sector has odd parity; this violates the symmetry principle that we discussed in Section 4 .

Let us turn our attention to the states with significant two particle content (marked by asterisks in Fig. 12). Surprisingly, adding extra gluons has only very little effect on the masses and structure functions of the lightest glueballs (typically only a few percent change). Similar observations had been made in $Q C D_{1+1}$ [11, 15. The reason for this suppression is not completely understood but has to do with the absence of UV divergences (the model is superrenormalizable) and IR divergences (which are screened in color singlet bound states) which normally drive the production of higher Fock components. In combination with the energy gap (due to the creation of an additional $\mathcal{J}_{z}=0$ glueball) that has to be overcome to mix in higher Fock components, these effects are very suppressed for low lying glueballs. Of course the situation changes when we examine heavier states. In that case there can be significant mixing of two and four particle wavefunctions.

An example of mixing of two and four particle wavefunctions is illustrated in Fig. 13. At $\mu^{2}=0$, the $2^{++}$sector of the theory has two nearly degenerate 


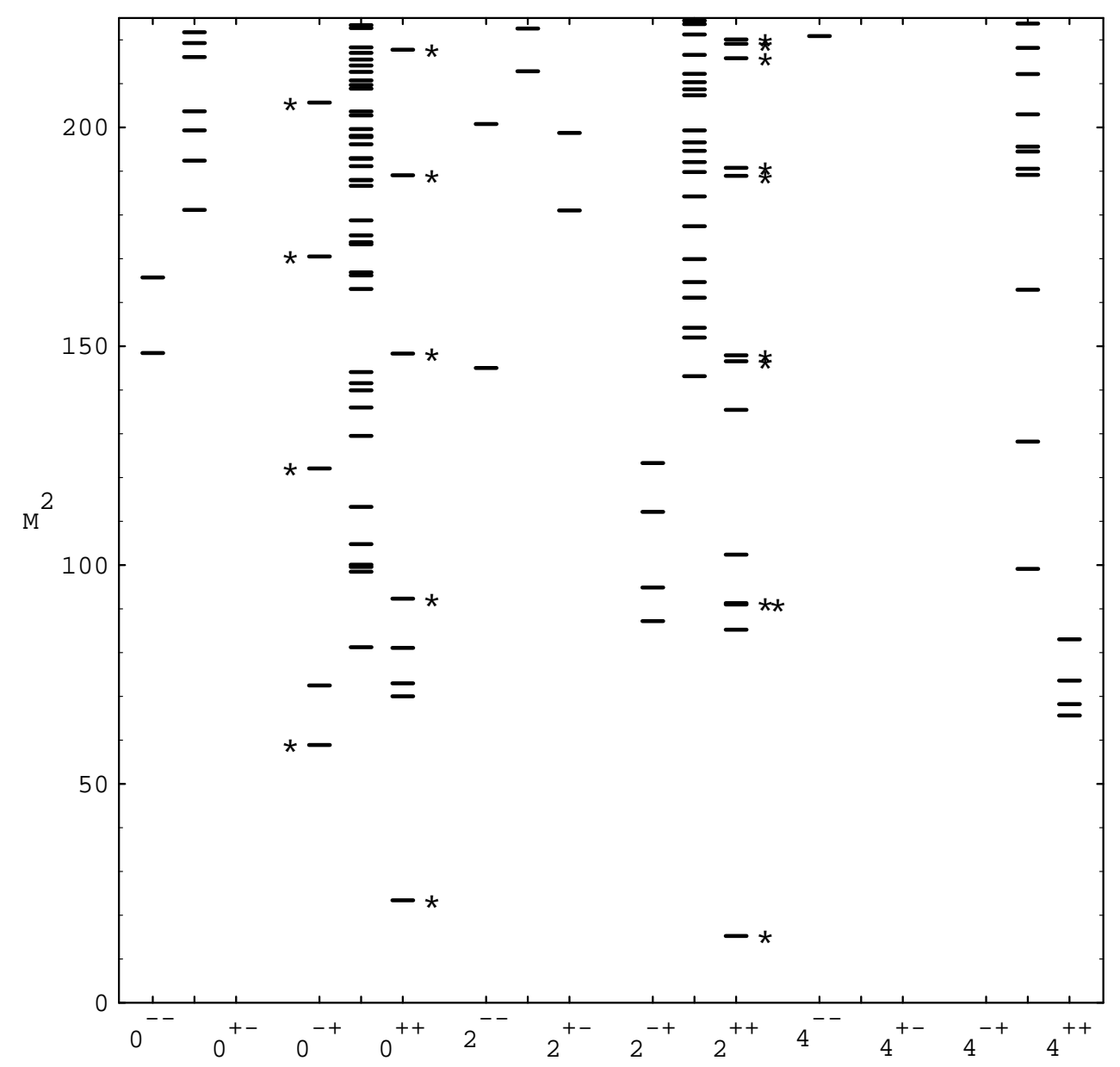

Figure 12: Four particle spectrum for various $\mathcal{J}_{z}{ }^{P C} ; K=12\left(\mathcal{J}_{z}=0,2\right)$ or $K=17\left(\mathcal{J}_{z}=4\right)$, and $\mu^{2}=0$. States whose parity could not be determined are placed midway between the corresponding $\mathcal{J}_{z}{ }^{+\mathcal{C}}$ and $\mathcal{J}_{z}{ }^{-\mathcal{C}}$ columns. States with more than $25 \%$ two particle content are marked with an asterisk. 


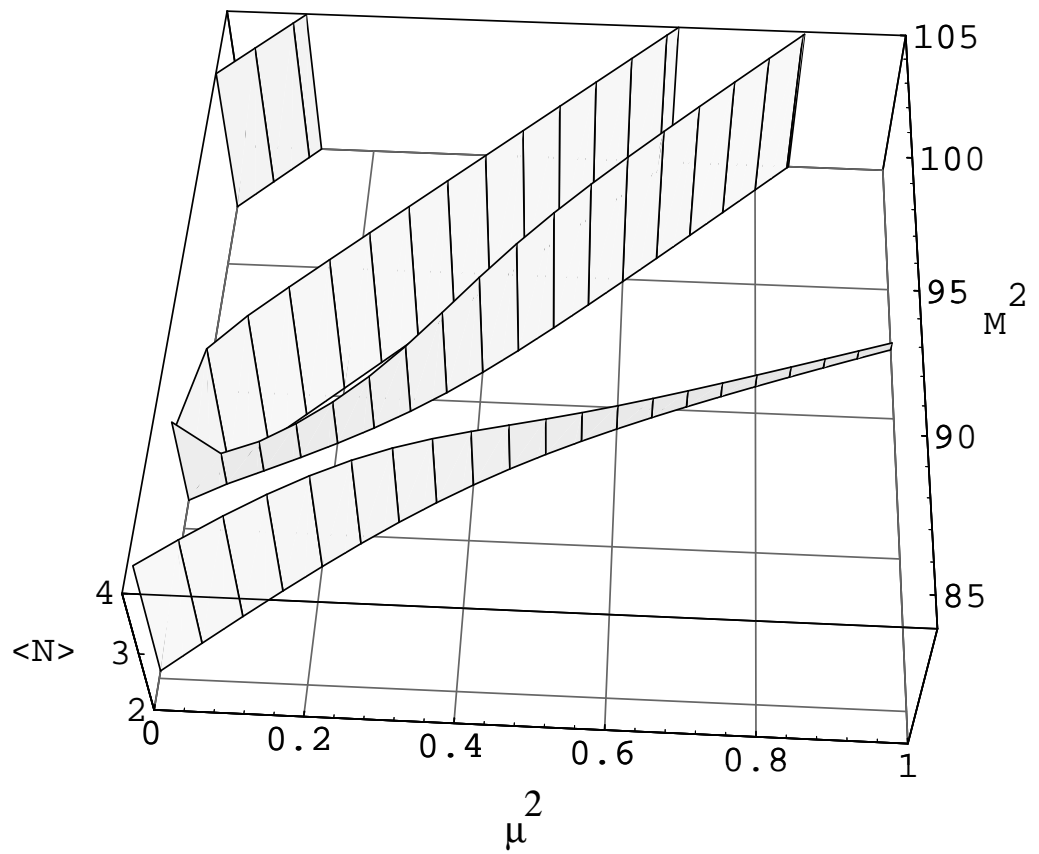

Figure 13: Expectation value of the number operator $\langle N\rangle$ vs $M^{2}$ and $\mu^{2}$ for the $2^{++}$sector of four particle spectrum, $K=12$. We see a classic example of level repulsion as the two and four particle states cross. 


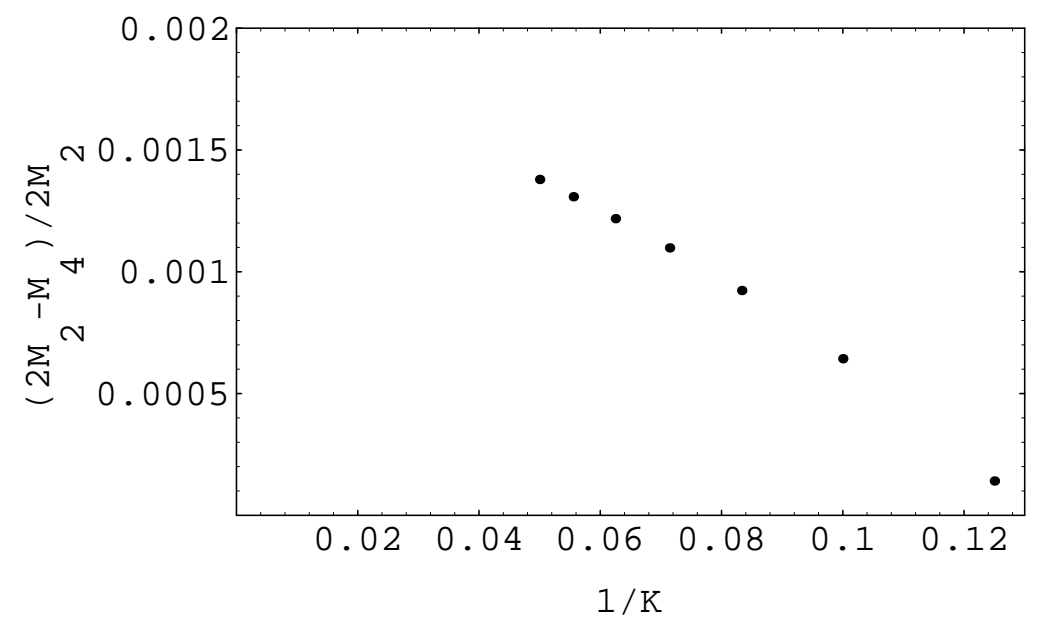

Figure 14: Binding energy of the lightest $4^{++}$glueball in units of the mass of two $2^{++}$glueballs as a function of the inverse harmonic resolution for $\mu^{2}=1$.

states at $M^{2}=91.0$ and 91.3. Both of these states have significant two particle content. In addition, there is a four particle state at $M^{2}=85.2$. As we increase $\mu^{2}$ to 1 , the two particle content of the two upper states decreases and the lower state becomes mainly two particles. Thus, we see a classic example of "level repulsion" as the two particle state crosses the four particle state. The strength of the interaction between the two and four particle states is roughly $\left(M^{2}\right)_{2,4} \approx 2$.

Finally, we consider the $\mathcal{C}=1$ states with predominately four particle content. Let us start with the lightest $4^{++}$glueball $\left(M^{2}=66\right)$ as an example. Numerical calculations at various values of the harmonic resolution $K$ (Fig. 14) show that its mass is slightly lower than the mass of two $2^{++}$glueballs; that is, decay into two widely separated $2^{++}$glueballs is energetically forbidden and the lowest $4^{++}$glueball is stable. In order to minimize numerical artifacts in the analysis of this state, we compare in the following the results for the $4^{++}$glueball taken at $K=20$ with those of the $2^{++}$glueball at half that value $(K=10)$. Furthermore, since no additional gluons (e.g. a 6 particle Fock component) were allowed, we compare with the $2^{++}$glueball restricted to the two gluon Fock sector.

Since its mass is extremely close to the mass of two $2^{++}$glueballs, one 


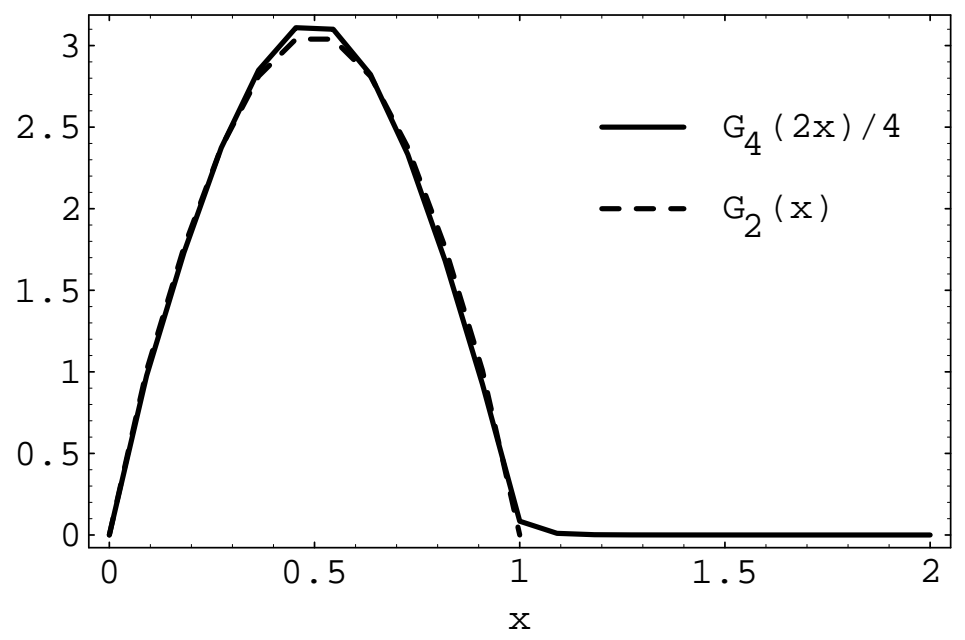

Figure 15: Gluon distributions for the $2^{++}$glueball (dashed curve, $K=10$, and $\mu^{2}=1$ ) and the $4^{++}$glueball (solid curve, $K=20$, and $\mu^{2}=1$ ). We have rescaled the $4^{++}$gluon distribution $G_{4}(x)$ such that it would yield the same distribution as the $2^{++}$glueball in the zero binding limit.

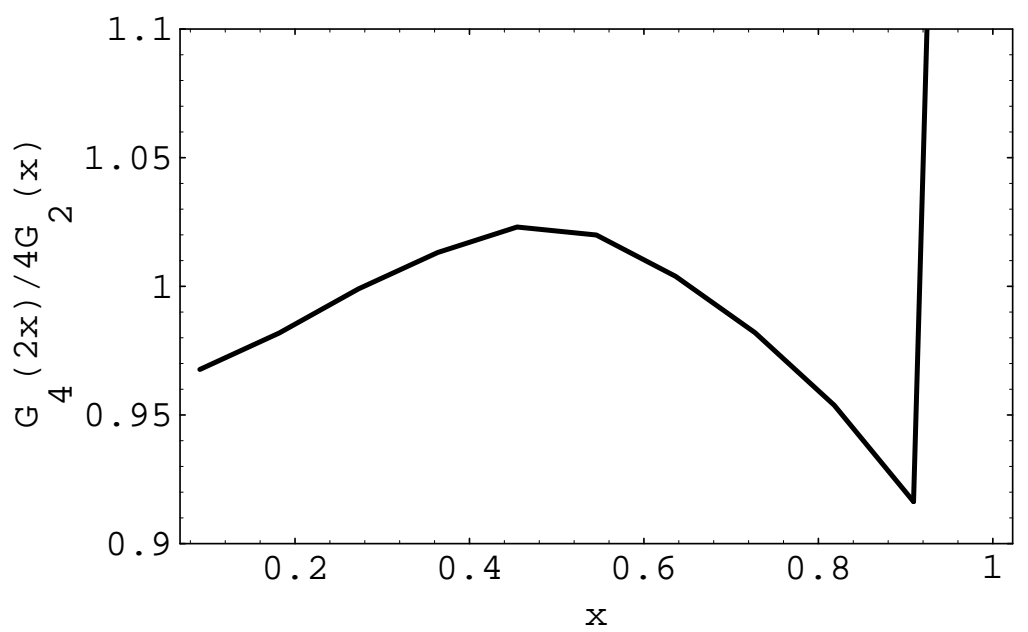

Figure 16: Ratio of the gluon distributions shown in Fig. 15. 
would expect that this state closely resembles a loosely bound pair of $2^{++}$ glueballs. A comparison of the gluon distributions in Figs. 15 and 16 confirms this effect since the distribution of the $2^{++}$and the (appropriately rescaled) $4^{++}$glueball are almost identical. The most pronounced differences are:

- a slight enhancement around $0.4<x<0.5$ which we interpret as a Bose-effect: when one brings two $2^{++}$glueballs together, there is an enhancement of the gluon distribution in the region of the maximum due to the symmetrization of the gluon wavefunction.

- a tail that goes beyond $x \geq 1$ in the case of the $4^{++}$glueball, which is kinematically forbidden for the $2^{++}$glueball. This behavior is also reflected in the divergence of the "EMC-ratio" at $x=1$ in Fig. 16.

- a depletion for $x<0.4$ and for $0.5<x<0.95$ : the momentum sum rule requires that the EMC-ratio drops below 1 at least once, which would "explain" the dip near $x=0.9$. However, we have not been able to find a similarly simple reason to explain the drop for $x<0.4$. See, however, the discussion of the deuteron in Ref. 15] which showed a similar behavior.

In summary, the $4^{++}$glueball has much in common with a "molecule" or an "atomic nucleus" in the sense that many of its properties can be explained by a picture of weakly bound constituents (here two $2^{++}$glueballs). Numerical limitations did not allow us to study whether similar bound states appear for $\mathcal{J}_{z}=6,8,10, \ldots$. Intuitively one would expect this to happen since (1) already weak attractive forces lead to binding in 1 spatial dimension and (2) gluons are bosons and one would not expect a shell structure such as the one which occurs in $Q C D_{1+1}$ [15].

We can apply a similar analysis to the $\mathcal{J}_{z}=2$ and $\mathcal{J}_{z}=0$ sectors of the four particle spectrum. We find that the lowest $2^{++}$glueball with mainly four particle content $\left(M^{2}=85\right)$ is a weakly bound state of a $0^{++}$and a $2^{++}$ glueball. Likewise the lowest $0^{++}$state with mainly four particle content $\left(M^{2}=70\right)$ consists of two weakly interacting $2^{++}$glueballs. However, in this case, the state is not bound. In fact, we can also identify the lowest $0^{++}$ state consisting of two weakly interacting $0^{++}$glueballs at $M^{2}=105$. This state is not bound either.

The excited states in the four particle spectrum consist mainly of pairs of weakly interacting glueballs which are moving at different relative momenta. 
In fact, with possibly one exception, all of the states below $M^{2}<140$ have this interpretation. For this reason, the levels shown in Fig. 12 are well approximated by the DLCQ formula for two free particles of invariant mass $M_{1}$ and $M_{2}$,

$$
M^{2}=K\left(\frac{M_{1}^{2}}{i}+\frac{M_{2}^{2}}{K-i}\right),
$$

and therefore the level spacing of these states is a finite $K$ artifact. The physically correct level spacing would be much smaller for the bound states and there would be a continuum spectrum in the $0^{++}$sector.

Because of this "continuum" of weakly interacting pairs of glueballs, it is somewhat difficult to distinguish any "true" four particle states. The only candidate that we could identify is a $0^{++}$state at $M^{2}=98$. If we were to take the $N \rightarrow \infty$ limit, this would be the lowest four particle state that would survive.

\section{Discussion}

How well does the tube model compare with experiment? The most reliable results that we have to compare to are from lattice gauge theory [16]. The most striking feature of our spectrum is the fact that the $2^{++}$glueball is lighter than the $0^{++}$glueball. This result is surely not correct. In principle, the coupling associated with the $\left[A_{r}, A_{s}\right]^{2}$ interaction in our Hamiltonian is independent of the coupling associated with the instantaneous interactions $P_{1}^{-}+\cdots+P_{4}^{-}$. Thus, for instance, one might adjust these couplings independently to obtain a spectrum that is more physically realistic 《4. However, we note two problems with this scheme. First, we point out the special behavior of the two particle spectrum at $\mu^{2}=0$ (namely, the constant wavefunction and zero eigenvalue) occurs only when the two couplings are identical. Moreover, in order to have better fit to lattice data, for instance, we would need to change the sign of the $\left[A_{r}, A_{s}\right]^{2}$ coupling. With such a sign change, the spectrum may be no longer be bounded from below. This is especially a concern if one attempts to adjust the couplings by fitting the lowest $0^{++}$and $2^{++}$glueballs to the lattice data.

Another concern is the slow convergence of the DLCQ calculations near $\mu^{2}=0$. The simplest improvement would be to choose antiperiodic instead of periodic boundary conditions for the gauge fields. However, if we later 
want to include fermions in the theory, we must choose periodic boundary conditions for the gauge fields. Another approach would be to solve the constraint equation for the zero mode of $A_{r}$ and substitute the result back into the Hamiltonian. This would produce new operators in the Hamiltonian and would substantially improve convergence to the continuum limit. This technique has already been demonstrated for $\phi^{4}$ theory in $1+1$ dimensions [17]. Finally, one can simply abandon DLCQ as a numerical technique and use a polynomial wave function basis instead; this is what we did for the two particle truncation. However, extending such a technique to a many particle calculation is relatively difficult.

In conclusion, we have made a thorough study of the $3+1$ dimensional tube model. In particular, we have examined the behavior of the $\mu^{2} \rightarrow 0$ limit and studied the convergence of the DLCQ calculations. In addition, we have successfully introduced an empirical technique to measure the parity of a state. We have found a discrete spectrum in the two and three particle sectors along with a continuous spectrum in the four particle sector and have studied the mixing of two and four particle states.

\section{Acknowledgements}

The authors would like to thank R. Bayer, S. Dalley, A. Kalloniatis, and H.C. Pauli for useful discussions and comments. M. Burkardt thanks the MPI für Kernphysik in Heidelberg for its hospitality. This work was supported, in part, by the Alexander Von Humboldt Stiftung.

\section{References}

[1] S. J. Brodsky, G. McCartor, H.-C. Pauli, and S. S. Pinsky, Particle World 3, 109 (1993).

[2] Bardeen, Pearson, and Rabinovici, Phys. Rev. D 21, 1037 (1980).

[3] S. Dalley and I. R. Klebanov, Phys. Rev. D 47, 2517 (1993); G. Bhanot, K. Demeterfi and I. R. Klebanov, Phys. Rev. D 48, 4980 (1993); K. Demeterfi and I. R. Klebanov, Nucl. Phys. B418, 15 (1994). 
[4] F. Antonuccio and S. Dalley, "Adjoint 2D QCD and Pure 3D QCD: A Comparison of Spectra" report No. OUTP-9518P and hep-lat/9505009; F. Antonuccio and S. Dalley, "Glueballs from 1+1 Dimensional Gauge Theories with Transverse Degrees of Freedom," report No. OUTP-9524P and hep-ph/9506456.

[5] H.-C. Pauli, A. Kalloniatis, and S. S. Pinsky, Phys. Rev. D 52, 1176 (1995); A. Kalloniatis, "On the zero modes and the vacuum problem: a study of scalar adjoint matter in Yang Mills theory via light-cone quantization," report No. MPI-H-V29-1995 and hep-th/9509027.

[6] M. Lüscher, Nucl. Phys. B219, 233 (1983); F. Lenz et al., Ann. Phys. (N. Y.) 208, 1 (1991); P. van Baal, Nucl. Phys. B369, 259 (1992); J. E. Hetrick, Int. J. Mod. Phys. A 9, 3153 (1994).

[7] H.-C. Pauli and R. Bayer, "Towards Solving QCD in Light-Cone Quantization - On the Spectrum of the Transverse Zero Modes for SU(2)," report No. MPI H-V32-1995 and hep-th/9510013.

[8] M. Engelhardt and B. Schrieber, Z. Phys. A351, 71 (1995).

[9] A. Kalloniatis and D. Robertson, Phys. Rev. D 50, 5262 (1994).

[10] H.-C. Pauli and S. J. Brodsky, Phys. Rev. D 32, 1993 (1985); H.-C. Pauli and S. J. Brodsky, Phys. Rev. D 32, 2001 (1985).

[11] K. Hornbostel, Ph. D Thesis, SLAC report No. SLAC-0333 (1988); K. Hornbostel, S. Brodsky, H.-C. Pauli, Phys. Rev. D 41, 3814 (1990).

[12] G. 't Hooft, Nucl. Phys. B72, 461 (1974); M. Einhorn, Phys. Rev. D 14, 3451 (1976).

[13] This is stated incorrectly in R. L. Jaffe and K. Johnson, Phys. Lett. 60B, 201 (1976).

[14] S. J. Brodsky, M. Burkardt, and I. Schmidt, Nucl. Phys. B441, 197 (1995).

[15] M. Burkardt, Nucl. Phys. A504, 762 (1989). 
[16] G.S. Bali et al., Phys. Lett. 309B, 378 (1993); H. Chen, J. Sexton, A. Vaccarino, and D. Weingarten, "The scalar and tensor glueballs in the valence approximation," report No. IBM-HET-94-1 and hep-lat/9401020.

[17] S. S. Pinsky, B. van de Sande, J. Hiller, Phys. Rev. D 51, 726 (1995). 